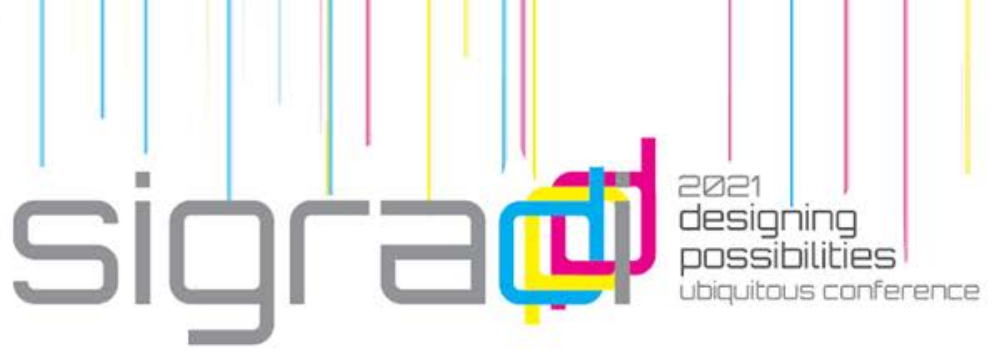

\title{
Interoperability Between Agisoft Metashape and Autodesk Revit Software
}

\author{
Gisele Martins, Giovana Ferreira, Gabriel Pazeti, Simone Helena Tanoue \\ Vizioli \\ Instituto de Arquitetura e Urbanismo (IAU USP) \\ giselewmartins@usp.br \\ giovana.fer@usp.br \\ gabrielp@usp.br \\ simonehtv@usp.br
}

\begin{abstract}
The 3D digital mapping to create models (Mesh) contributes to the documentation of the architectural heritage, by the accuracy of the details achieved. The documentation through Building Information Modelling (BIM) technologies is a current strategy to digitize the as-built information. Despite the potential for integrated use of these technologies, interoperability between them presents a gap involving complexity and operability in the work process. This research aims to investigate the interface and communication capabilities between Mesh models, generated by photogrammetry, and a BIM model, through the interoperability between Agisoft Metashape and Autodesk Revit software. By analyzing a new format for heritage documentation through digital technologies, using as object of study the façade of the Centro de Divulgação Científica e Cultural (CDCC-USP) in São Carlos, the results show that the joint use of technologies does not present great losses of information, unlike the techniques that are currently used.
\end{abstract}

Keywords: Interoperability, Photogrammetry, Mesh, RPAS, HBIM.

\section{Introdução}

Este trabalho é parte de um projeto de pesquisa do grupo N.ELAC do Instituto de Arquitetura e Urbanismo (IAU USP). Tecnologias inovadoras incorporadas à Arquitetura, se revelam por meio de softwares que potencializam o processo projetivo. Aliado às premências da conservação do patrimônio cultural, vive-se hoje um momento de constante desenvolvimento de tecnologias expressado por métodos e técnicas no contexto da cultura digital. Estas, por sua vez, se 
mostram capazes de auxiliar no processo de documentação, preservação e gestão patrimonial. Os procedimentos tradicionais ocorrem de forma complexa, envolvendo vários agentes especialistas, em decorrência das características dos objetos a serem registrados, dos diferentes requisitos de qualidade e de resolução. A seleção das tecnologias (hardware e software), a determinação dos procedimentos, workflows apropriados e a constatação de que os produtos finais apresentam semelhança em relação às especificações técnicas, representam dificuldade (Stylianidis; Patias; Quinteiro, 2011).

Encontrou-se nas tecnologias de sensoriamento remoto e varredura uma alternativa para os métodos de levantamento manuais, já que possibilitam reunir grande densidade de informações de forma rápida, compilando com precisão a configuração real dos objetos, contendo suas irregularidades e imperfeições decorrentes do processo construtivo, e as deformações e desgastes decorrentes do ciclo de vida da edificação (Groetelaars; Amorim, 2012). Segundo Simon, 2000, a fotogrametria vem em auxílio da preservação, para preencher a lacuna entre a documentação, planejamento e implementação de novas intervenções na arquitetura, independente da característica conservadora ou inovadora. Os modelos Mesh gerados pela fotogrametria (Agisoft Metashape) apresentam riquezas de detalhes e texturas. A partir de métodos de varredura, como a fotogrametria digital, o DSM (Dense Stereo Matching) e o 3D Laser Scanning, é possível extrair informações de maneira rápida e precisa, produzindo a modelagem de nuvens de pontos e modelos Mesh por processamento digital utilizando o software Agisoft Metashape, por exemplo. Incorpora-se aos recursos da tecnologia o uso de Remotely Piloted Aircraft System (RPA), os popularmente chamados drones, que possuem diversas vantagens por permitirem uma documentação sem perdas de qualidade em regiões de difícil acesso e com extrema velocidade de varredura de áreas extensas como sítios arqueológicos, fachadas e detalhes de objetos (Campos, Cattani, da Silva, 2020).

A produção de documentação por meio de tecnologias como as plataformas Building Information Modelling (BIM) mostra-se como uma estratégia atual para digitalizar as informações referentes ao as-built. O principal avanço em um modelo BIM é a parametrização e a incorporação de informações ao modelo, nível de Informação (Lol). Tem-se utilizado modelos Mesh, gerados a partir de mapeamento digital 3D, para remodelagem geométrica manual em softwares de modelos BIM, como o Autodesk Revit e esse processo além de moroso, apresenta perdas de informação e representação (Manferdini, Remondino, 2012; Dezen-Kempter et al., 2015; Tang et al., 2010).

Existem no mercado programas capazes de criar um modelo Mesh dos mapeamentos tridimensionais, como por exemplo, o Blender, 3D Studio Max e Sketchup. A questão é que esses aplicativos impossibilitam a inserção de informações e parametrizações, ou seja, o modelo é somente visual gráfico 


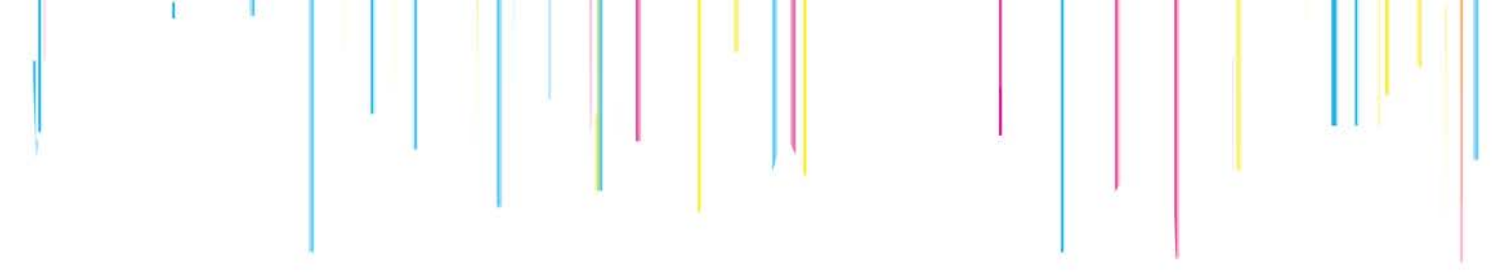

3D. Mesh é um conceito para descrever e gerar geometria 3D em um computador; já o BIM é uma tecnologia para produzir informações geométricas, documentação, coordenação, e é capaz de criar modelos Mesh, contudo, produzir geometria é apenas uma parte própria desta tecnologia. Embora o software fotogramétrico e o BIM usem diferentes tipos de tecnologias para desenvolver o Mesh, a abordagem da geometria é diferente.

De acordo com o guia de implementação BIM inglês (PAS 1192-2, 2013), modelos BIM podem apresentar diferentes níveis de desenvolvimento e os termos encontrados para eles são: $L O D$ (nível de desenvolvimento), $L O D$ (nível de detalhe gráfico) e $L O I$ (nível de informação). O nível de desenvolvimento é o termo mais geral e é menos útil para acordos detalhados e divide-se em $L O D$ e $L O I$. Uma família com um nível ou detalhe $(L O D)$ baixo, por exemplo, possui uma forma geométrica simples, como um cubo ou cilindro, e não contém nenhum material especificado. O LOI é usado para indicar o nível de informação vinculado a uma família, desempenhando um papel importante em um modelo BIM. Uma família com um nível muito alto de informações inclui, por exemplo, folhas de dados específicas do fabricante e instruções de manutenção.

Desenvolvimento, Detalhe ou Informação é o quão básico para complexo os modelos BIM são construídos. Podendo variar do nível 100, básico para as 3 categorias até 500 , onde o modelo apresenta mais detalhes gráficos e informações. Os três conceitos funcionam juntos e não como uma entidade independente, porém o LOI é o parâmetro que controla se a geometria é uma caixa pura ou mais detalhada, interferindo no LoD e no LOD. A imagem (Figura 1 e 2) apresentada na sequência exemplifica as diferenças entre os três termos.

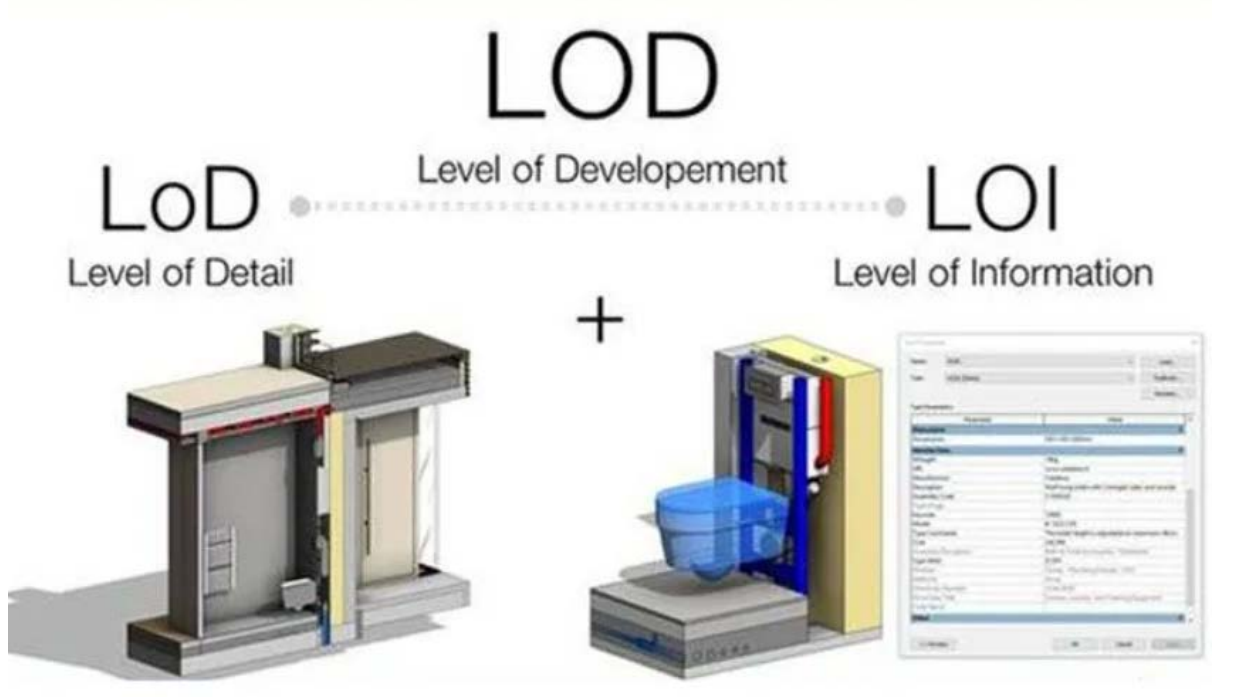




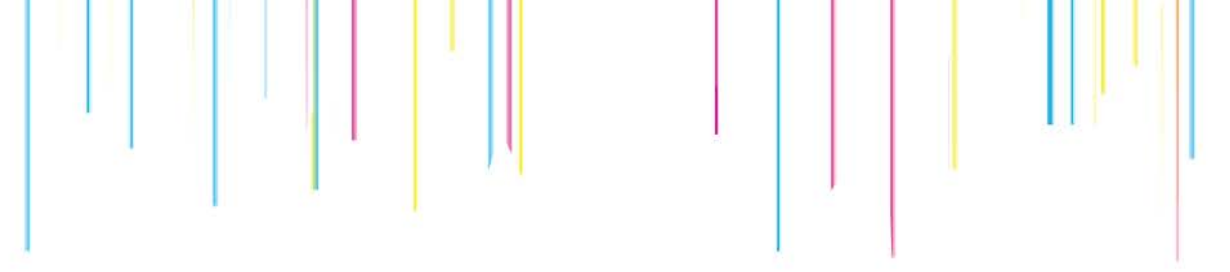

Figura 1. LOD e LOI. Fonte: SPBIM, 2020.
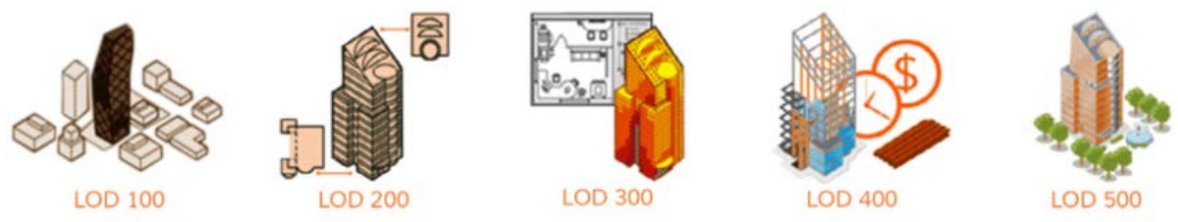

Figura 2. Nível de Desenvolvimento LOD. Fonte: SPBIM, 2020.

Segundo Groetelaars e Amorim (2012), uma questão importante no emprego do paradigma do BIM para representar e gerenciar edificações existentes está relacionada ao modo como as informações geométricas (formas e dimensões) podem ser capturadas e introduzidas em uma ferramenta para a geração do modelo. Apesar dos benefícios apontados na utilização dessas metodologias, Tang et al. (2010) alertam sobre a criação do modelo BIM realizado por meio do resultado da digitalização do edifício existente por nuvem de pontos, ainda ser um processo fundamentalmente manual, moroso, de natureza subjetiva e sujeito a erros. Esta integração ainda motiva a necessidade de ferramentas de automação ou, pelo menos, semiautomatizadas de reconhecimento de componentes visando a modelagem.

Ainda em Dezen-Kempter, Soibelman et al., 2015, concluem que os desafios específicos à engenharia reversa diante das abordagens para o reconhecimento dos objetos utilizados para a modelagem as-built baseada em tecnologia BIM, diante de dados capturados pelas tecnologias de varredura, ainda não tem uma solução.

As dificuldades de operacionalidade dos modelos gerados a partir da fotogrametria e os modelos BIM, os quais requerem hardwares com maior memória e velocidade ao processarem modelos tridimensionais altamente detalhados está na interoperabilidade entre os mesmos, resultando em alguns casos, em modelos de alta resolução que demandam muita memória no hardware. Podemos dizer então que apesar do reconhecimento dos potenciais de utilização integrada dessas tecnologias (fotogrametria e BIM), a interoperabilidade entre as mesmas apresenta uma lacuna envolvendo a complexidade e a operacionalidade no processo de trabalho. A relevância desta pesquisa se dá no potencial de inovação tecnológica ao estabelecer relação entre estes métodos computacionais utilizando a exploração na comunicação de dois softwares específicos. 


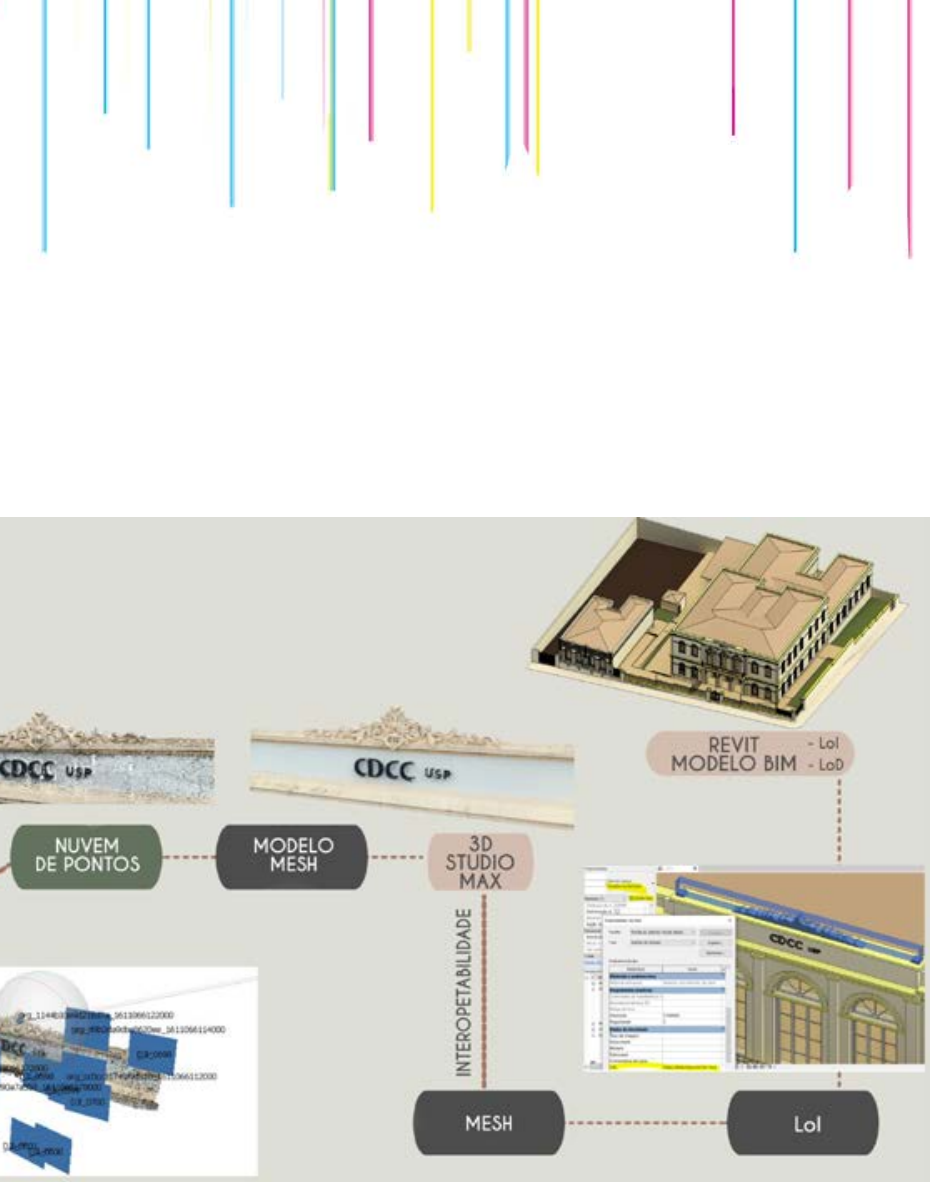

Figura 5. Desenvolvimento proposto na pesquisa .Fonte: autores, 2021.

\section{$4 \quad$ Resultados e Discussão}

A utilização conjunta das duas tecnologias permite uma documentação em níveis de detalhamentos superiores às atuais documentações. O modelo BIM é limitado quanto ao desenho preciso de ornamentos complexos, além de exigir boas configurações de hardware, contudo com o uso da fotogrametria, torna-se possível com a inserção das informações construtivas do patrimônio e de detalhamentos complexos dos ornamentos e texturas verossímeis dentro do nível de informação $(L O I)$ do Revit contornando o uso de hardwares custosos. De acordo com o quadro 1 apresentado na sequência foi possível verificar as diferentes representações gráficas nos modelos.

\begin{tabular}{|c|c|c|}
\hline & DETALHE ORNAMENTO & FACHADA \\
\hline $\begin{array}{c}\text { Modelo } \\
\text { BIM }\end{array}$ & CDCC usp & \\
\hline
\end{tabular}




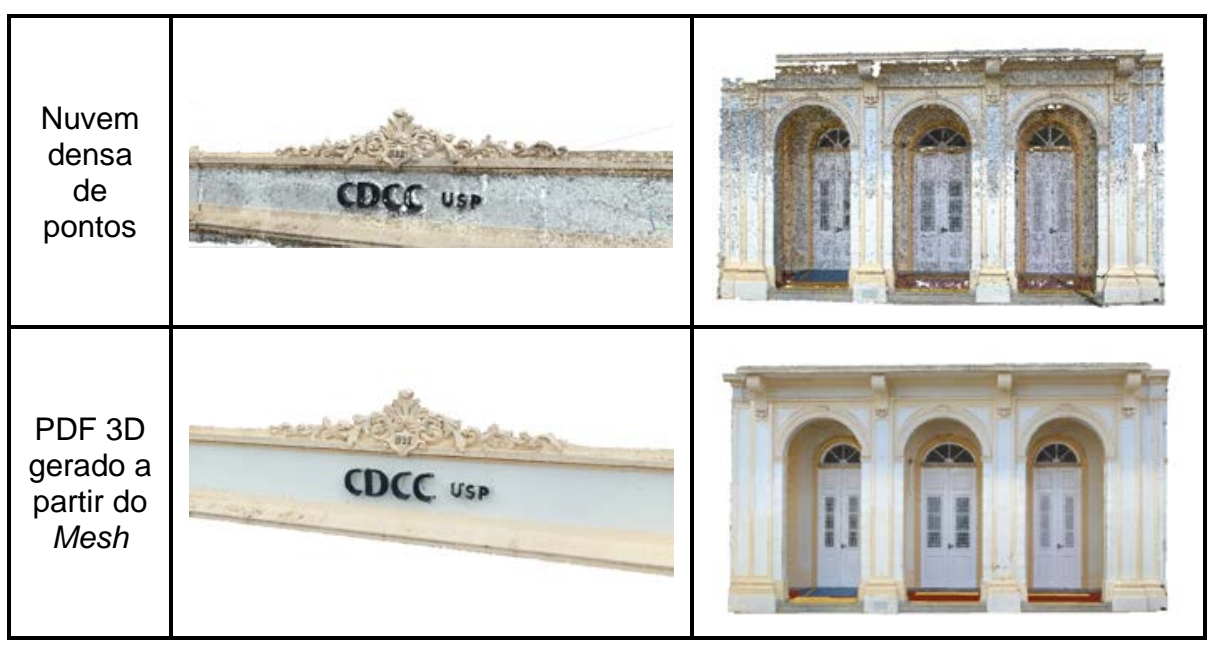

Quadro 1. Comparação entre os modelos BIM, a nuvem de pontos e o resultado final em PDF 3D gerado a partir do FBX. Fonte: autores, 2021.

O quadro 2 apresenta as vantagens e desvantagens do uso do procedimento de interoperabilidade utilizado atualmente de forma manual e o processo proposto na pesquisa:

\begin{tabular}{|c|c|c|c|}
\hline \multicolumn{2}{|c|}{$\begin{array}{c}\text { PROCESSO UTILIZADO } \\
\text { ATUALMENTE }\end{array}$} & \multicolumn{2}{|c|}{$\begin{array}{c}\text { PROCESO PROPOSTO NA } \\
\text { PESQUISA }\end{array}$} \\
\hline $\begin{array}{c}\text { Modelo BIM produzido manualmente } \\
\text { a partir do Mesh gerado por meio da } \\
\text { fotogrametria }\end{array}$ & $\begin{array}{c}\text { Modelo BIM com modelos Mesh } \\
\text { gerados por meio fotogrametria em } \\
\text { LOI e LoD }\end{array}$ \\
\hline Vantagens & Desvantagens & Vantagens & Desvantagens \\
\hline $\begin{array}{c}\text { Fidelidade gráfica } \\
\text { dos modelos BIM } \\
\text { em relação ao } \\
\text { real }\end{array}$ & $\begin{array}{c}\text { Dificuldade em } \\
\text { extrair textura e } \\
\text { detalhes }\end{array}$ & $\begin{array}{c}\text { Visualização de } \\
\text { texturas e } \\
\text { detalhes }\end{array}$ & $\begin{array}{c}\text { Menor nível de } \\
\text { detalhes gráficos no } \\
\text { modelo BIM }\end{array}$ \\
\hline & $\begin{array}{c}\text { Processo moroso e } \\
\text { manual }\end{array}$ & $\begin{array}{c}\text { Inserção direta } \\
\text { do modelo } \\
\text { Mesh (sem } \\
\text { redesenho) }\end{array}$ & $\begin{array}{c}\text { Não possui o modelo } \\
\text { gráfico BIM total } \\
\text { detalhado e com } \\
\text { textura real }\end{array}$ \\
\hline & $\begin{array}{c}\text { Tamanho do } \\
\text { arquivo }\end{array}$ & $\begin{array}{c}\text { Arquivo mais } \\
\text { leve }\end{array}$ & \\
\hline
\end{tabular}




\begin{tabular}{|l|l|c|l|}
\hline & $\begin{array}{c}\text { Associar } \\
\text { detalhes reais } \\
\text { às informações } \\
\text { do modelo } \\
\text { (LOI) }\end{array}$ & \\
\hline & & $\begin{array}{c}\text { Compreensão } \\
\text { detalhada e } \\
\text { verossímil }\end{array}$ & \\
\hline
\end{tabular}

Quadro 2. Comparação entre os processos. Fonte: autores, 2021.

\section{Conclusão}

Os resultados consistiram em vincular informações tridimensionais (modelos Mesh em extensão PDF 3D) aos modelos BIM, por meio da inserção no nível de informação $L O I$ de uma modelagem BIM no Revit. Pode-se dizer que o LOI do modelo BIM do Revit ganhou um alto nível e o modelo Mesh gerado a partir da fotogrametria contribuiu com mais informações e detalhes gráficos. Sendo assim, o tempo e esforço para a modelagem gráfica do modelo $B I M$ no campo do $L O D$ foi menor e não necessitou de tantos detalhes gráficos. Por meio da conjunção das modelagens, de maneira pragmática, constata-se que o processo não apresenta grandes perdas da informação.

\section{Agradecimentos}

À Pró-Reitoria de Pesquisa, de Ensino e de Extensão pela concessão de bolsas PUBs; ao IAU.USP pela infraestrutura e apoio; ao N.ELAC pelos equipamentos; à CPG (Comissão de Pós-Graduação) pelo auxílio financeiro para inscrição no evento científico; à equipe de design computacional; à Diretoria do CDCC, Profa. Salete Linhares Queiroz e Profa. Nelma Regina Segnini Bossolan, pelo trabalho conjunto; à Mayara Capistrano Costa Fook, que forneceu o modelo BIM; e à FAPESP, projeto no. 2018/18958-0.

\section{Referências}

Campos, S., Cattani, A., \& Silva, F. (2020). Geração de conteúdo em realidade aumentada com o uso de drones na digitalização 3D por fotogrametria, caso da igreja do desterro em são luís maranhão, Brasil. In G. Nunez \& G. Oliveira (Orgs.). Design em pesquisa (vol 3, pp. 534-545). Marcavisual. 
